\section{Relapses and treatment-related events contributed equally to poor prognosis in children with ABL-class fusion positive B-cell acute lymphoblastic leukemia treated according to AIEOP-BFM protocols}

Gunnar Cario, ${ }^{1 *}$ Veronica Leoni, ${ }^{2 *}$ Valentino Conter,${ }^{2 *}$ Andishe Attarbaschi, ${ }^{3}$ Marketa Zaliova, ${ }^{4}$ Lucie Sramkova, ${ }^{4}$ Gianni Cazzaniga, ${ }^{2}$ Grazia Fazio, ${ }^{2}$

Rosemary Sutton, ${ }^{5}$ Sarah Elitzur, ${ }^{6}$ Shai Izraeli, ${ }^{6}$ Melchior Lauten, ${ }^{7}$

Franco Locatelli, ${ }^{8}$ Giuseppe Basso, ${ }^{9}$ Barbara Buldini, ${ }^{9}$ Anke K. Bergmann, ${ }^{10}$ Jana Lentes,${ }^{10}$ Doris Steinemann, ${ }^{10}$ Gudrun Göhring, ${ }^{10}$

Brigitte Schlegelberger ${ }^{10}$ Oskar A. Haas, ${ }^{3}$ Denis Schewe, ${ }^{1}$

Swantje Buchmann, ${ }^{1}$ Anja Moericke, ${ }^{1}$ Deborah White, ${ }^{11}$ Tamas Revesz, ${ }^{12}$

Martin Stanulla, ${ }^{13}$ Georg Mann, ${ }^{3}$ Nicole Bodmer ${ }^{14}$ Nira Arad-Cohen, ${ }^{15}$

Jan Zuna, ${ }^{4}$ Maria Grazia Valsecchi, ${ }^{2}$ Martin Zimmermann, ${ }^{13}$

Martin Schrappe ${ }^{1 *}$ and Andrea Biondi ${ }^{2 t}$

${ }^{1}$ Pediatrics, University Hospital Schleswig-Holstein, Campus Kiel, Kiel, Germany; ${ }^{2}$ Clinica Pediatrica and Centro Ricerca Tettamanti, Università di Milano-Bicocca, Fondazione MBBM/ S.Gerardo Hospital, Monza, Italy; ${ }^{3}$ St. Anna Kinderspital and Children's Cancer Research Institute, Vienna, Austria; ${ }^{4} \mathrm{CLIP}$, Department of Pediatric Hematology and Oncology, $2^{\text {nd }}$ Faculty of Medicine, Charles University and University Hospital Motol, Prague, Czech Republic; ${ }^{5}$ Molecular Diagnostics, Children's Cancer Institute, University of NSW, Sydney, NSW, Australia; ${ }^{6}$ Pediatric Hematology-Oncology, Schneider Children's Medical Center, Petah Tikva, and Sackler Faculty of Medicine, Tel Aviv University, Israel; ${ }^{7}$ Pediatrics, University Hospital Schleswig-Holstein, Campus Lübeck, Lübeck, Germany; ${ }^{8}$ Department of Pediatric Hematology and Oncology, Scientific Institute for Research and Healthcare (IRCCS) Childrens' Hospital Bambino Gesù, Sapienza, University of Rome, Rome, Italy; 'IIGM Torino and Pediatric Hemato-Oncology, SDB Departiment, University of Padova, Padova, Italy; ${ }^{10}$ Department of Human Genetics, Medical School Hannover, Hannover, Germany; ${ }^{11}$ Cancer Theme, South Australian Health and Medical Research Institute, Adelaide, Australia; ${ }^{12}$ Women's and Children's Hospital, SA Pathology, University of Adelaide, Adelaide, Australia; ${ }^{13}$ Department of Pediatric

Hematology/Oncology, Hannover Medical School, Hannover, Germany; ${ }^{14}$ University Children's Hospital Zurich, Zurich, Switzerland and ${ }^{15}$ Pediatric Hematology-Oncology Department, Ruth Rappaport Children's Hospital, Rambam Health Care Campus, Rappaport Faculty of Medicine, Technion-Israel Institute of Technology, Haifa, Israel

*GC, VL and VC contributed equally as co-first authors.

"MS and AB contributed equally as co-senior authors.

\section{ABSTRACT}

A BL-class fusions other than $B C R-A B L 1$ characterize around $2-3 \%$ of precursor B-cell acute lymphoblastic leukemia. Case series indicated that patients suffering from these subtypes have a dismal outcome and may benefit from the introduction of tyrosine kinase inhibitors. We analyzed clinical characteristics and outcome of 46 ABL-class fusion positive cases other than $B C R$-ABL1 treated according to AIEOP-BFM (Associazione Italiana di Ematologia-Oncologia Pediatrica-Berlin-FrankfurtMünster) ALL 2000 and 2009 protocols; 13 of them received a tyrosine kinase inhibitor (TKI) during different phases of treatment. ABL-class fusion positive cases had a poor early treatment response: minimal residual disease levels of $\geq 5 \times 10^{-4}$ were observed in $71.4 \%$ of patients after induction treatment and in $51.2 \%$ after consolidation phase. For the entire cohort of 46 cases, the 5 -year probability of event-free survival was $49.1+8.9 \%$ and that of overall survival $69.6+7.8 \%$; the cumulative incidence of relapse was $25.6+8.2 \%$ and treatment-related mortality (TRM) $20.8+6.8 \%$. One out of 13 cases with TKI added to chemotherapy relapsed while eight of 33 cases
Ferrata Storti Foundation

\section{Correspondence:}

GUNNAR CARIO

gunnar.cario@uksh.de

Received: July 8, 2019.

Accepted: October 10, 2019.

Pre-published: October 10, 2019.

doi:10.3324/haematol.2019.231720

Check the online version for the most updated information on this article, online supplements, and information on authorship \& disclosures: www.haematologica.org/content/105/7/1887

\section{(C)2020 Ferrata Storti Foundation}

Material published in Haematologica is covered by copyright. All rights are reserved to the Ferrata Storti Foundation. Use of published material is allowed under the following terms and conditions:

https://creativecommons.org/licenses/by-nc/4.0/legalcode. Copies of published material are allowed for personal or internal use. Sharing published material for non-commercial purposes is subject to the following conditions:

https://creativecommons.org/licenses/by-nc/4.0/legalcode, sect. 3. Reproducing and sharing published material for commercial purposes is not allowed without permission in writing from the publisher. 
without TKI treatment suffered from relapse, including six in 17 patients who had not received hematopoietic stem cell transplantation. Stem cell transplantation seems to be effective in preventing relapses (only three relapses in 25 patients), but was associated with a very high TRM (6 patients). These data indicate a major need for an early identification of ABL-class fusion positive acute lymphoblastic leukemia cases and to establish a properly designed, controlled study aimed at investigating the use of TKI, the appropriate chemotherapy backbone and the role of hematopoietic stem cell transplantation. (Registered at: clinicaltrials.gov identifier: NTC00430118, NCT00613457, NCT01117441).

\section{Introduction}

Continuous optimization of risk-adapted multi-agent treatment has led to excellent curative rates in the majority of children and adolescents suffering from acute lymphoblastic leukemia (ALL). ${ }^{1.9}$ However, the progress in ALL subtype classification according to the nature of specific sentinel genetic aberrations identified molecular ALL subgroups like low-hypodiploid, KMT2A-rearranged or $B C R-A B L 1$ positive precursor-B-ALL (B-ALL) with distinct biological and clinical characteristics associated with poor outcome. Intensive chemotherapy, including allogeneic hematopoietic stem cell transplantation (HSCT) for some of these patients, is associated with severe toxicity and long-term sequelae.

In this context, one of the first ALL genetic aberrations identified was the gene fusion $B C R-A B L 1$ resulting from the chromosomal translocation $\mathrm{t}(9 ; 22)$ (generating the socalled Philadelphia chromosome), translated into the BCRABL1 fusion protein, a constitutively active tyrosine kinase, which can be inhibited by tyrosine kinase inhibitors (TKI). This is an excellent example for a successful molecular treatment target: the addition of the firstgeneration TKI imatinib to intensive chemotherapy backbone has led, in fact, to a significant improvement of outcome in children with Philadelphia chromosome positive ALL $\left(\mathrm{Ph}^{+}\right.$ALL) with cure rates of $60-70 \% \cdot{ }^{10-18}$ The Children's Oncology Group (COG) studies showed a clear advantage in $\mathrm{Ph}^{+}$ALL from continuous protracted exposure to TKI combined with chemotherapy, challenging the indications to transplant for all patients with $\mathrm{Ph}^{+}$ALL. ${ }^{16-18}$ COG results were confirmed by the European intergroup study group for treatment of $\mathrm{Ph}^{+}$ALL (EsPhALL) in the EsPhALL2004 and the subsequent EsPhALL2010 studies, showing that intensive chemotherapy combined with imatinib given continuously from induction phase allows a remarkable reduction in the rate of HSCT, without affecting outcome. ${ }^{13-15}$ However, these trials also demonstrated that the combination of chemotherapy and imatinib is associated with a high rate of treatment-related toxicity and mortality.

In the last decade, different tyrosine kinase gene fusions other than $B C R$ - $A B L 1$ have been identified which are sensitive to TKI similar as $B C R-A B L 1$. These so called ABLclass fusions typically comprise rearrangements of the $A B L 1, A B L 2, P D G F R B$ and CSF1R genes, each of which can have different fusion partner genes. ABL-class fusion positive B-ALL subtypes other than BCR-ABL1 have been identified showing a gene expression profile largely similar to that of $\mathrm{Ph}^{+} \mathrm{ALL}$. Therefore, they are included in the $B C R$-ABL1-/Ph-like-ALL group recognized as a provisional entity in the 2016 World Health Organization classification of myeloid neoplasms and acute leukemia although they only make up a minor proportion of patients in this new category. Whereas BCR-ABL1-/Ph-like-ALL accounts for $15-20 \%$ of all pediatric B-ALL, the frequency of ABLclass fusion positive B-ALL is estimated to be about $2-3 \%$ which is similar to the frequency of $B C R-A B L 1$ pos. ALL. ${ }^{19}$ ${ }^{21} B C R-A B L 1$-/Ph-like ALL is associated with other highrisk clinical features, such as older age, elevated white blood cell (WBC) count at diagnosis, high rates of endinduction minimal residual disease (MRD), as well as increased risk of induction failure and of leukemia relapse. ${ }^{22-28}$

Data on the ABL-class fusion positive ALL other than $\mathrm{Ph}^{+} \mathrm{ALL}$ are rare and are limited to anecdotal case reports. In this study, we retrospectively analyzed clinical characteristics and outcome of ABL-class fusion positive cases treated based on contemporary MRD-based protocols of the Associazione Italiana di Ematologia-Oncologia Pediatrica-Berlin-Frankfurt-Münster (AIEOP-BFM ALL study group. The aim was to provide a comprehensive picture of current outcome of these cases without the addition of a TKI to chemotherapy, and to get first data on those cases in which a TKI was added to chemotherapy. It should serve as a basis on which to decide whether the addition of a TKI to chemotherapy may be beneficial, when taking into account the risk of a relevant increase of toxicity and treatment-related mortality (TRM).

\section{Methods}

\section{Patients and diagnostics}

This retrospective survey of ABL-class fusion positive B-ALL other than $\mathrm{Ph}^{+}$ALL was performed in patients aged 1-17 years, treated from October 2000 to August 2018 according to the AIEOP-BFM ALL 2000 and 2009 protocols in Centers in Austria, Australia, Czech Republic, Germany, Israel, Italy, and Switzerland.

Routine diagnostics was performed according to national standards based on protocol requirements. ${ }^{8,929-31}$ Diagnosis of ALL was made when $25 \%$ or more lymphoblastic cells were present cytomorphologically in the bone marrow. Flow-cytometry immunophenotyping was performed based on the AIEOP-BFM consensus guidelines. ${ }^{29}$ Complete remission (CR) was defined as the absence of physical signs of leukemia or detectable leukemia cells on blood smears, a bone marrow with active hematopoiesis and $<5 \%$ blasts, and morphologically normal cerebrospinal fluid. Presence of ETV6-RUNX1, BCR-ABL1 and KMT2A-AFF1 fusion transcripts was screened as previously described. 8,9

ABL-class fusions screening, not required by protocols, was performed in a minority of patients according to the policy of individual centers or due to poor response to treatment. Methods used included fluorescence in situ hybridization (FISH, e.g. using probes by Cytocell $^{\circledR}$, Cambridge, UK), multiplex or singleplex reversetranscription polymerase chain reaction (PCR), ${ }^{32}$ array comparative genomic hybridization $(\mathrm{CGH})$ (Agilent Technologies, Waldbronn, Germany) with subsequent confirmation by panel- 
A

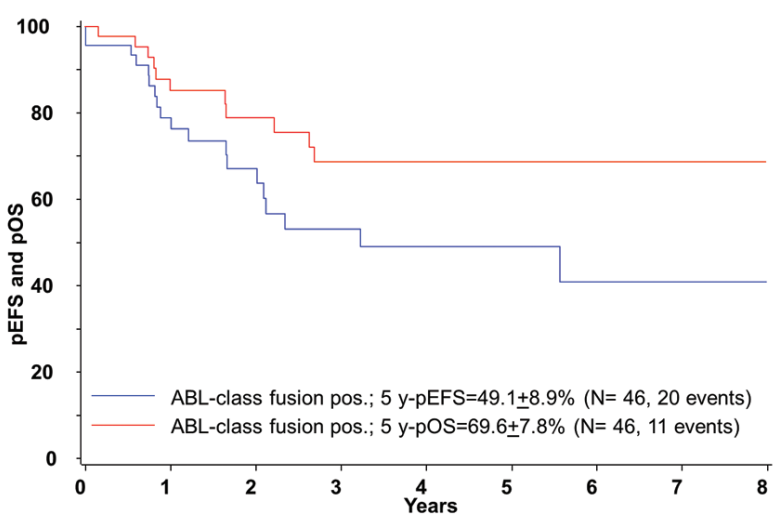

C

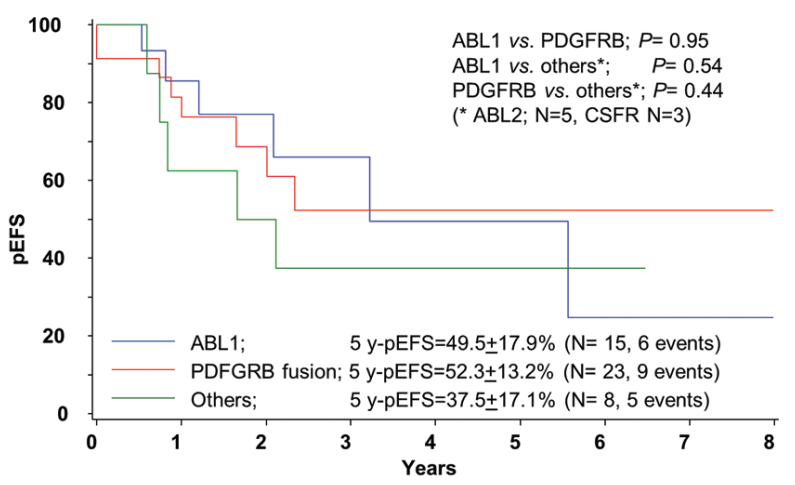

B

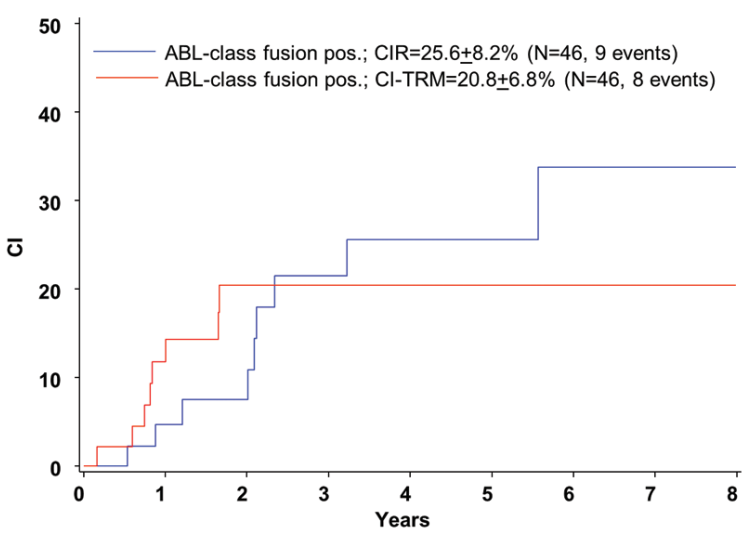

D

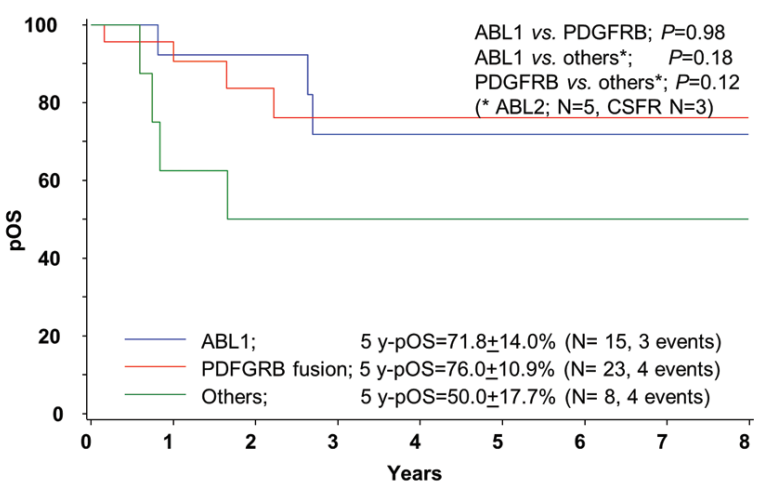

Figure 1. Treatment outcome of patients with pediatric ABL-class fusion positive acute lymphoblastic leukemia (ALL). Kaplan-Meier estimates for the whole cohort of 46 cases. (A) Event-free survival (pEFS) and overall survival (pOS) at 5 years (y). (B) Cumulative incidence of relapses (CIR) and of treatment-related mortality (ClTRM) at 5 years. According to ABL-class fusion subtype, $A B L 1$, PDGFRB, others (ABL2 $\mathrm{n}=5, C S F R \mathrm{n}=2$ ): (C) EFS. (D) OS.

based RNA-sequencing (e.g. TruSight RNA Pan-Cancer Panel; Illumina, San Diego, CA, USA), whole transcriptome or direct panel-based RNA-sequencing.

In both protocols, patient stratification was mainly based on quantitative assessment of minimal residual disease (MRD) using clone-specific immunoglobulin- and T-cell receptor-gene rearrangements by PCR (PCR-MRD) after induction (treatment day 33) and consolidation (day 78) therapy. In AIEOP-BFM ALL 2009, MRD was additionally measured by flow cytometry on treatment day 15 (FCM-MRD) ${ }^{33}$ The logistics of the AIEOP-BFM ALL studies, cell sample isolation, and MRD marker identification, as well as MRD-based risk stratification of the AIEOP-BFM ALL 2000 study, have been previously reported..$^{8,9}$ In the AIEOP-BFM ALL 2009 trial, patients were additionally allocated to the highrisk (HR) group, when PCR-MRD was $\geq 5 \times 10^{-4}$ on day 33 and still measurable on day 78 and/or if FCM-MRD at day 15 was $\geq 10 \%$.

Details on chemotherapy regimens and randomized treatment interventions, as well as HSCT indication, were reported elsewhere. ${ }^{8,9,34}$ The study AIEOP-BFM ALL 2000 is registered at Www.clinicaltrials.gov by BFM as NCT00430118 and by AIEOP as NCT00613457; the study AIEOP-BFM ALL 2009 is registered as NCT01117441. The analyses were approved by local Institutional Review Boards and informed consent was obtained from the patients and/or guardians in accordance with the Declaration of Helsinki.

\section{Statistical analysis}

Event-free survival (EFS) was calculated from diagnosis to first failure, which was defined as death during induction therapy, resistance, relapse, death in $\mathrm{CR}$, or development of a second malignant neoplasm (SMN). Rates were calculated according to Kaplan-Meier and compared by log-rank test. ${ }^{35,36}$ Kaplan-Meier plots that compared HSCT with chemotherapy were adjusted to account for the waiting time to transplantation (with a landmark at median time to HSCT). Cumulative incidence of relapse and TRM functions were constructed by the method of Kalbfleisch and Prentice and compared with Gray test. ${ }^{37,38}$ Proportional differences between patient groups were analyzed by $\chi^{2}$ or Fisher's exact tests.

\section{Results}

We identified 46 ABL-class fusion positive cases diagnosed between October 2000 and August 2018 treated according to AIEOP-BFM ALL 2000 and 2009 protocols.

$A B L 1$ fusions were identified in 15 cases, $A B L 2$ fusions in five cases, CSF1R fusions in three cases, and PDGFRB rearrangements in 23 cases (Online Supplementary Table S1).

Overall, 33 patients received chemotherapy without the addition of any TKI (no-TKI group); a TKI was added on an individual basis and not according to protocol during treatment in 13 cases (TKI group; imatinib in 8 and dasatinib in 5 cases) diagnosed between February 2011 and April 2018. In eight of these 13 cases, TKI was introduced at the end of induction or during consolidation therapy, in four during post-consolidation HR-blocks and in one case 
after HSCT (for details see Online Supplementary Figure S1).

Altogether, 36 of $46(78.3 \%)$ patients were treated in the HR group [(no-TKI 24 of $33(72.7 \%)]$, TKI 12 of 13 $(92.3 \%))$, and HSCT in first CR was performed in 25 of 46
(54.3\%) patients: in 16 of $33(48.5 \%)$ of no-TKI and in 9 of $13(69.3 \%)$ of TKI-treated patients (Table 1$)$. Compared to the entire group of B-ALL patients of the AIEOP-BFM ALI 2000 study, ABL-class fusion positive cases were older and

Table 1. Patients' and clinical characteristics and response to treatment according to ABL-class fusion and tyrosine kinase inhibitor (TKI) treatment in comparison with the entire AIEOP-BFM 2000 B-ALL cohort.

\begin{tabular}{|c|c|c|c|c|c|c|c|c|c|c|}
\hline \multirow{2}{*}{$\begin{array}{c}\text { ABL-class } \\
\text { fusion pos } \\
\text { cases } \\
\text { Total } \\
\text { n (\%) }\end{array}$} & \multicolumn{4}{|c|}{ Treatment without additional TKI ${ }^{1}$} & \multicolumn{4}{|c|}{ Treatment with additional TKI } & \multirow{2}{*}{$\begin{array}{c}\text { ALL-BFM } \\
2000 \text { B-ALL } \\
\text { Total } \\
\text { n (\%) }\end{array}$} & \multirow[t]{2}{*}{$P^{2}$} \\
\hline & $\begin{array}{c}\text { All } \\
n(\%)\end{array}$ & $\begin{array}{c}\text { PDGFRB } \\
\text { fusion } \\
n(\%)\end{array}$ & $\begin{array}{c}\text { ABL1 } \\
\text { fusion } \\
n(\%)\end{array}$ & $\begin{array}{c}\text { Others }^{3} \\
\text { n (\%) }\end{array}$ & $\begin{array}{c}\text { All } \\
n(\%)\end{array}$ & $\begin{array}{c}\text { PDGFRB } \\
\text { fusion } \\
\text { n (\%) }\end{array}$ & $\begin{array}{c}\text { ABL1 } \\
\text { fusion } \\
n(\%)\end{array}$ & $\begin{array}{c}\text { Others } \\
\text { n (\%) }\end{array}$ & & \\
\hline
\end{tabular}

$\begin{array}{lllllllllll}\text { N. of patients } \quad 46(100.0) & 33(100.0) & 17(100.0) & 10(100.0) & 6(100.0) & 13(100.0) & 6(100.0) & 5(100.0) & 2(100.0) & 3854(100.0)\end{array}$

\begin{tabular}{llllllllllll} 
Sex & & & & & & & n.s. \\
Male & $29(63.0)$ & $22(66.7)$ & $12(70.6)$ & $7(70.0)$ & $3(50.0)$ & $7(53.8)$ & $3(50.0)$ & $3(60.0)$ & $1(50.0)$ & $2062(53.5)$ & \\
Female & $17(37.0)$ & $11(33.3)$ & $5(29.4)$ & $3(30.0)$ & $3(50.0)$ & $6(46.2)$ & $350.0)$ & $2(40.0)$ & $1(50.0)$ & $1792(46.5)$ & \\
\hline Age at diagnosis (years) & & & & & & & & & & & $<.0001$ \\
$\quad 0-9$ & $22(47.8)$ & $15(45.5)$ & $7(41.2)$ & $7(70.0)$ & $1(16.7)$ & $6(46.2)$ & $1(16.7)$ & $4(80.0)$ & $1(50.0)$ & $2999(77.8)$ \\
$>10$ & $24(52.2)$ & $18(54.5)$ & $10(58.8)$ & $3(30.0)$ & $5(83.3)$ & $7(53.8))$ & $5(83.3)$ & $1(20.0)$ & $1(50.0)$ & $855(22.2)$
\end{tabular}

\begin{tabular}{|c|c|c|c|c|c|c|c|c|c|c|}
\hline WBC count $^{4}\left(x 10^{9} / L\right)$ & & & & & & & & & & \\
\hline Lower than 50 & $16(34.8)$ & $14(42.4)$ & $8(47.1)$ & $4(40.0)$ & $2(33.3)$ & $2(15.4)$ & $2(33.3)$ & 0 & 0 & $3288(85.3)$ \\
\hline $50-<100$ & $9(19.6)$ & 7 (21.2) & $4(23.5)$ & $2(20.0)$ & $1(16.7)$ & $2(15.4)$ & $1(16.7)$ & $1(20.0)$ & 0 & $325(8.4)$ \\
\hline 100 or higher & 19 (41.3) & $12(36.4)$ & $5(29.4)$ & $4(40.0)$ & $3(50.0)$ & $7(53.8)$ & $3(50.0)$ & $3(60.0)$ & $1(50.0)$ & $241(6.3)$ \\
\hline No information & $2(4.3)$ & 0 & 0 & 0 & 0 & $2(15.4)$ & 0 & $1(20.0)$ & $1(50.0)$ & 0 \\
\hline
\end{tabular}

\begin{tabular}{|c|c|c|c|c|c|c|c|c|c|c|c|}
\hline \multirow{2}{*}{\multicolumn{11}{|c|}{ NCI Risk group 5}} & \\
\hline & & & & & & & & & & & \multirow[t]{4}{*}{$<.0001$} \\
\hline SR & $7(15.2)$ & $7(21.2)$ & $3(17.6)$ & $3(30.0)$ & $1(16.7)$ & 0 & 0 & 0 & & $2574(66.8)$ & \\
\hline HR & $37(80.4)$ & $26(78.8)$ & $14(82.4)$ & $7(70.0)$ & $5(83.3)$ & $11(84.6)$ & $6(100.0)$ & $4(80.0)$ & $1(50.0)$ & $1280(33.2)$ & \\
\hline No information & $2(4.3)$ & 0 & 0 & 0 & 0 & $2(15.4)$ & 0 & $1(20.0)$ & $1(50.0)$ & 0 & \\
\hline Pred. response ${ }^{6}$ & & & & & & & & & & & $<.0001$ \\
\hline Good & $22(47.8)$ & $17(51.5)$ & $5(29.4)$ & $9(90.0)$ & $3(50.0)$ & $5(38.5)$ & $1(16.7)$ & $3(60.0)$ & $1(50.0)$ & 3619 (93.9) & \\
\hline Poor & $23(50.0)$ & $16(48.5)$ & $12(70.6)$ & $1(10.0)$ & $3(50.0)$ & $7(53.8)$ & $5(83.3))$ & $1(20.0)$ & $1(50.0)$ & $214(5.6)$ & \\
\hline No information & $1(2.2)$ & 0 & & 0 & 0 & $1(7.7)$ & 0 & $1(20.0)$ & 0 & $21(0.5)$ & \\
\hline $\mathrm{MRD}^{7}$ at End of induction & & & & & & & & & & & $<.0001$ \\
\hline$\geq 5 \times 10^{-2}$ & $22(47.8)$ & $17(51.5)$ & $13(76.5)$ & $1(10.0)$ & $3(50.0)$ & $5(38.5)$ & $4(66.6)$ & $1(20.0)$ & & $86(2.2)$ & \\
\hline $5 \times 10^{-4}-<5 \times 10^{-2}$ & $8(17.4)$ & $5(15.2)$ & $2(11.8)$ & $3(30.0)$ & 0 & $3(23.1)$ & $1(16.7)$ & $1(20.0)$ & $1(50.0)$ & $610(15.8)$ & \\
\hline$<5 \times 10^{4}$ & $12(26.1)$ & $9(27.3)$ & $1(5.9)$ & $6(60.0)$ & $2(33.3)$ & $3(23.1)$ & 0 & $3(60.0)$ & 0 & $2925(75.9)$ & \\
\hline No information & $4(8.7)$ & $2(6.1)$ & $1(5.9)$ & 0 & $1(16.7)$ & $2(15.4)$ & $1(16.7)$ & 0 & $1(50.0)$ & $233(6.0)$ & \\
\hline MRD after consolidation & & & & & & & & & & & $<.0001$ \\
\hline$\geq 5 \times 10^{-2}$ & $9(19.6)$ & $8(24.2)$ & $5(29.4)$ & $1(10.0)$ & 20 & $1(7.7)$ & 0 & $1(20.0)$ & 0 & $34(0.9)$ & \\
\hline $5 \times 10^{-4}-<5 \times 10^{-2}$ & $12(26.1)$ & $9(27.3)$ & $8(47.1)$ & 0 & $1(16.7)$ & $3(23.1)$ & $2(33.3)$ & 0 & $1(50.0)$ & $151(3.9)$ & \\
\hline$<5 \times 10^{4}$ & $20(43.5)$ & $15(45.5)$ & $3(17.6)$ & $9(90.0)$ & $3(33.3)$ & $5(38.5)$ & $2(33.3)$ & $3(60.0)$ & & $3452(89.6)$ & \\
\hline No information & $5(10.9)$ & $1(3.0)$ & $1(5.9)$ & 0 & 0 & $4(30.8)$ & $2(33.3)$ & $1(20.0)$ & $1(50.0)$ & $217(5.6)$ & \\
\hline Treatment group & & & & & & & & & & & $<.0001$ \\
\hline SR & $3(6.5)$ & $3(9.1)$ & 0 & $2(20.0)$ & $1(16.7)$ & 0 & 0 & 0 & 0 & 1337 (34.7) & \\
\hline IR & $5(10.9)$ & $4(12.1)$ & 0 & $3(30.0)$ & $1(16.7)$ & $1(7.7)$ & 0 & $1(20.0)$ & 0 & $2088(54.2)$ & \\
\hline HR & $36(78.3)$ & $24(72.7)$ & $17(100.0)$ & $3(30.0)$ & $4(66.7)$ & $12(92.3)$ & $6(100.0)$ & $4(80.0)$ & $2(100.0)$ & $429(11.1)$ & \\
\hline No information & $2(4.3)$ & $2(6.1)$ & 0 & $2(20.0)$ & 0 & 0 & 0 & 0 & & 0 & \\
\hline $\mathrm{HSCT}^{8}$ & & & & & & & & & & & $<.0001$ \\
\hline No & $21(45.7)$ & $17(51.5)$ & $5(29.9)$ & $10(100.0)$ & $2(33.3)$ & $4(30.7)$ & $1(16.7)$ & $3(60.0)$ & 0 & 3684 (95.1) & \\
\hline Yes & $25(54.3)$ & $16(48.5)$ & $12(70.1)$ & 0 & $4(66.7)$ & $9(69.3)$ & $5(83.3)$ & $2(40.0)$ & $2(100.0)$ & $216(4.9)$ & \\
\hline
\end{tabular}

ALL: acute lymphoblastic leukemia. ${ }^{~}$ TKI: tyrosine kinase inhibitor. ${ }^{2}$ Fisher test comparing all ABL-class positive cases to the B-ALL AIEOP-BFM 2000 cohort, patients with no information excluded from test. ${ }^{3}$ Others: ABL2 $\mathrm{n}=5, \mathrm{CSFR} 1 \mathrm{n}=3 .{ }^{4} \mathrm{WBC}$ : white blood cell count. ${ }^{5} \mathrm{NCI}-\mathrm{SR}, \mathrm{WBC}<50 \mathrm{x} 10^{9} / \mathrm{L}$ and age $<10$ years; NCI-HR, WBC $>50 \mathrm{x} 10^{\circ} / \mathrm{L}$ and/or age $>10$ years ${ }^{6} \mathrm{Good}:<1,000$ leukemic blood blasts / $\mu \mathrm{L}$ on treatment day 8, poor: more than $1000 / \mu \mathrm{L} .{ }^{7} \mathrm{MRD}$ : minimal residual disease. ${ }^{8} \mathrm{HSCT}$ : hematopoietic stem cell transplantation. 
had higher white blood cell counts at diagnosis (WBC), with a statistically significant difference for NCI-HR features $(P<0.0001$ each) (Table 1$)$. ABL-class fusion positive cases had a significantly worse response to treatment compared to the entire B-ALL 2000 cohort: prednisone poor response (PPR) was observed in $50 \%$ versus $5.6 \%$ of patients with data available, high MRD level $\left(25 \times 10^{-4}\right)$ after induction treatment (EoI) in $71.4 \%$ versus $19.2 \%$ and after consolidation (EoC) in $51.2 \%$ versus $5.1 \%$ of cases with data available. There are, however, differences by type of ABL-class fusion: the majority of PDGFRB-fusion positive cases with data available showed a PPR (17 of 23, $73.9 \%$ ), high EoI-MRD (20 of 21, $95.2 \%$ with data available) as well as high EoC-MRD (15 of 20,75\% with data available). In contrast, in ABL1-class positive cases, PPR (2 of 14, 14.3\%), high EoI-MRD (6 of 15, 40.0\%), and high EoC-MRD (2 of 14, 14.3\%) were observed much less frequently (Table 1). Of note, we observed a favorable MRD response (MRD negative or low-positive) at EoC in 5 of 6 ABL-class positive cases with MRD data available in whom a TKI was added to chemotherapy before EoC (three had an ABL1-fusion and two had a PDGFRB-fusion).

\section{A}

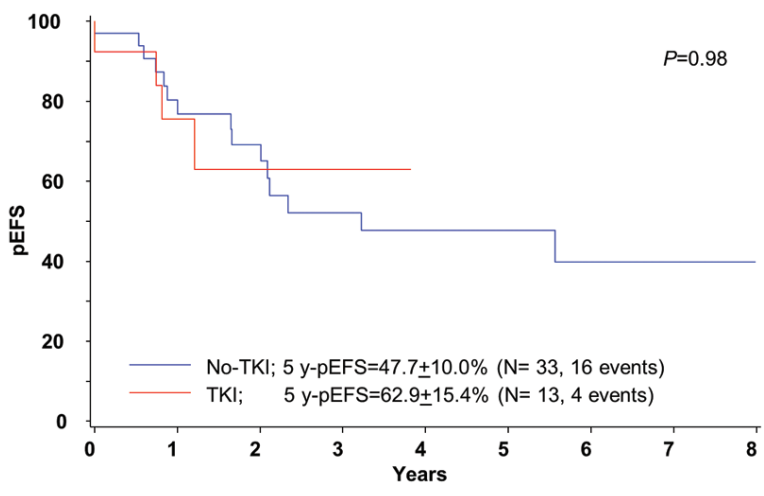

For the entire cohort of 46 cases, 5-year EFS was $49.1+8.9 \%$ and 5 -year OS $69.6+7.8 \%$; CIR was $25.6+8.2 \%$ (9 events) and TRM 20.8+6.8\% (8 events) (Figure 1A and B). Six of the nine patients with leukemia relapse in first CR were successfully treated subsequently and are in long-term CR. One case was resistant to treatment (resistance defined as blast persistence after three HR blocks) and two presented with SMN after HSCT (one thyroid cancer and one lymphoma), both patients with SMN were treated successfully. Details of the events are shown in Table 2.

No significant differences in 5-year EFS and 5-year OS were observed comparing the ABL-class subgroups $A B L 1$-fusions, PDGFRB-fusions and other fusions (5 ABL2 and 3 CSFR1 fusions) (Figure 1C and D). Interestingly, the ABL1-fusion positive group, which showed a better treatment sensitivity as compared to the PDGFRB-fusion positive group, had a higher frequency of relapses (5 of 15 patients vs. 3 of 23); of note HSCT was performed only in 2 of 15 ABL1-fusion positive cases versus 17 of 23 PDGFRB-fusion positive cases (Online Supplementary Figure S2A-C).

Patients treated with TKI had worse features (12 of 13

B

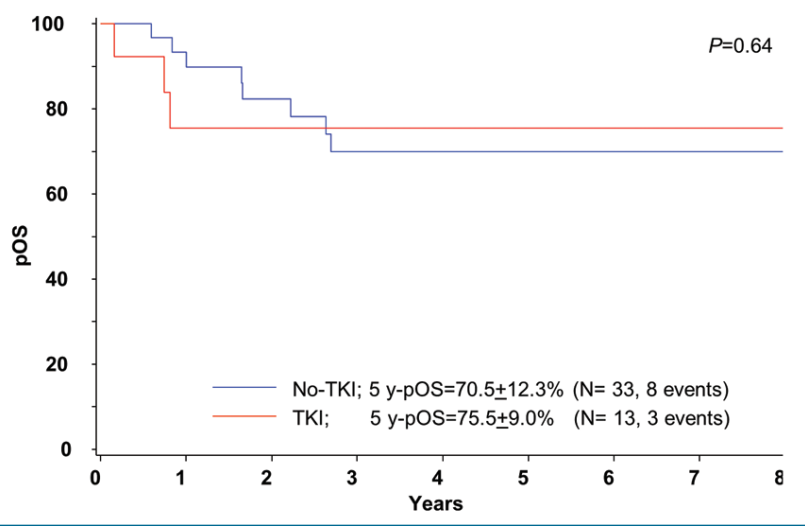

Figure 2. Treatment outcome of patients with pediatric ABL-class fusion positive acute lymphoblastic leukemia (ALL) according to treatment without or with tyrosine kinase inhibitor (TKI). Kaplan-Meier estimates comparing patients treated without TKI (no-TKI) and with TKI (TKI) are shown. (A) Event-free survival (EFS) at 5 years (y). (B) Overall survival (OS) at 5 years.

Table 2. Distribution of events according to tyrosine kinase inhibitor (TKI) treatment.

\begin{tabular}{lccc} 
& $\begin{array}{c}\text { Total } \\
n(\%)\end{array}$ & $\begin{array}{c}\text { No TK } 1^{1} \\
n(\%)\end{array}$ & $\begin{array}{c}\text { TK } \\
n(\%)\end{array}$ \\
All cases & $46(100.0)$ & $33(100.0)$ & $13(100.0)$ \\
Resistant $^{2}$ & $1(2.2)$ & $1(3.0)$ & 0 \\
Relapses $^{3}$ & $9(19.6)$ & $8(24.2)^{4}$ & $1(7.7)^{5}$ \\
After chemotherapy $_{\text {After HSCT }}$ & $6(13.0)$ & $6(18.1)$ & 0 \\
Death in induction & $3(6.5)$ & $2(6.1)$ & $1(7.7)$ \\
Death in CR & $1(2.2)$ & $5(15.2)$ & $2(15.4)$ \\
After chemotherapy & $7(15.2)$ & 0 & $1(7.7)$ \\
After HSCT & $1(2.2)$ & $5(15.2)$ & $1(7.7)$ \\
SMN $^{8}$ & $6(13.0)$ & $2(6.1)$ & 0 \\
\hline
\end{tabular}

ALL: acute lymphoblastic leukemia. ${ }^{~ T K I}$ : tyrosine kinase inhibitor. ${ }^{2}$ Resistant patients are those who did not achieve complete remission (CR) by end of the third high-risk (HR) block of chemotherapy. ${ }^{3}$ Relapses: 5 cases ABL1 fusion positive (pos.), 3 cases PDGFRB fusion pos., 1 case CSF1R fusion pos. ${ }^{4}$ No-TKI relapses: after chemotherapy: 1 very early 3 early, 2 very late (both RCSD11-ABL1 pos.), after hematopoietic stem cell transplantation (HSCT): 4 months and 17 months after HSCT. ${ }^{5}$ TKI relapse: 1 case 9 months post HSCT. ${ }^{6}$ HSCT. ${ }^{7} \mathrm{CR}$ : complete remission. ${ }^{8}$ SMN: second malignant neoplasms: 1 case with post-transplant lymphoma, 1 case with thyroid cancer after HSCT. 
A

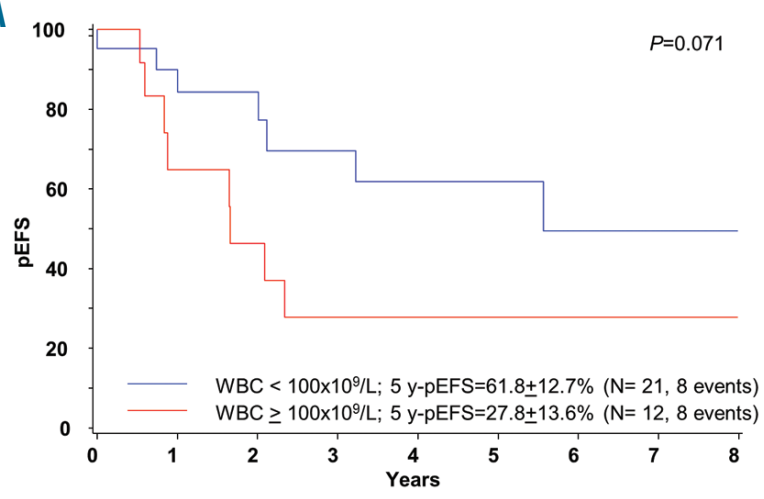

B

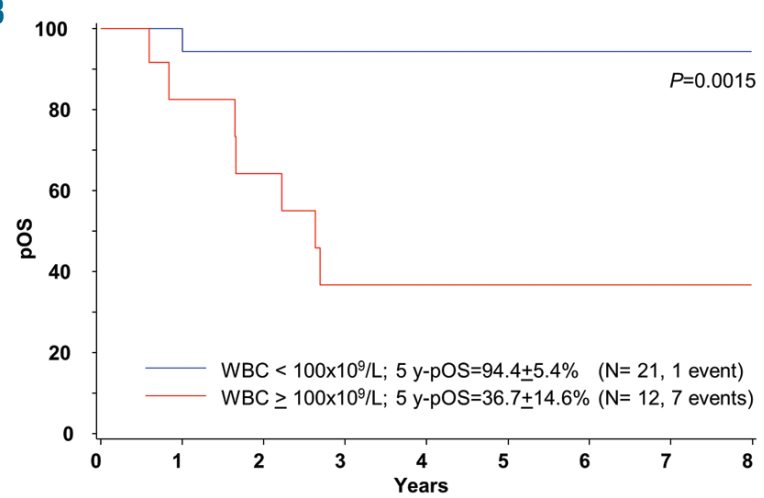

Figure 3. Treatment outcome of patients with pediatric ABL-class fusion positive acute lymphoblastic leukemia (ALL) according to white blood cell count at diagnosis (WBC). Kaplan-Meier estimates comparing patients with WBC $<100 \times 10^{\circ} / \mathrm{L}$ and patients with WBC equal or $>100 \times 10^{\circ} / \mathrm{L}$ are shown. (A) Event-free survival (pEFS) at 5 years. (B) Overall survival (pOS) at 5 years.

were HR vs. 24 of 33 in no-TKI treated patients) and the majority of them underwent HSCT (9 of 13 vs. 16 of 33); their 5-year EFS and 5-year-OS did not differ significantly compared with the no-TKI group: EFS no-TKI $47.7+10.0 \%$, TKI $62.9+5.4 \%, \quad P=0.98 ; \quad$ OS no-TKI $70.9+9.0 \%$, TKI $75.5+12.3 \%, P=0.64$ (Figure 2A and B). Whereas TRM was similar in both groups, only one out of 13 cases of the TKI patients relapsed versus 8 of 33 of no-TKI cases (Online Supplementary Figure S3A and B). Of note, there were four late occurring events in the no-TKI group: two SMN after HSCT and two late relapses in ZMIZ1-ABL1 positive patients not transplanted.

No significant difference in outcome between patients treated with or without HSCT were seen: 5-year EFS was $47.9+11.4 \%$ versus $55.0+15.5 \%, \quad P=0.35 ; 5$-year OS $66.7+10.5 \%$ versus $84.0+10.6 \%, \quad P=0.22$ ) (Online Supplementary Figure $S 4 A$ and $B$ ). However, analyzing the events in more detail, it is remarkable that the majority of events in HSCT-treated patients $(n=25)$ were non-relapse events (TRM=6, $S M N=2$, relapses $=3$ ) whereas in patients not transplanted $(\mathrm{n}=21)$ relapses predominated (TRM $=1$, relapses $=6$ ) (Table 2). In those patients who were not treated with TKI, the CIR and TRM rate in transplanted versus not transplanted cases were $13.2+9.3 \%$ versus $43.8+17.8 \%, P=0.06$ (CIR) and $32.3+12.4 \%$ versus $0.0 \%$, $P=0.034$ (TRM) (Online Supplementary Figure S4C and D).

Analysis by MRD showed a 5 -year EFS of $40.4 \pm 11.4 \%$ in EoI-MRD $\geq 5 \times 10^{-4}$ versus $76.2 \pm 14.8 \%$ in EoI $\mathrm{MRD}<5 \times 10^{-4}, P=0.11$ ) (Online Supplementary Figure S5A); CIR was similar in both MRD groups $(27.3+11.2 \%$ vs. $23.8+15.9 \%, P=0.74)$, while TRM in patients with EoI-MRD $\geq 5 \times 10^{-4}$ was $25.4+9.4 \%$ versus $0.0 \%$ in EoI $M R D<5 \times 10^{-4}(P=0.1)$ (Online Supplementary Figure S5B). By EoC-MRD, 5-year EFS in cases with an $M R D \geq 5 \times 10^{-4}$ was $46.2+12.1 \%$ versus $56.7+15.4 \%$ in those with $\mathrm{MRD}<5 \times 10^{-4}, P=0.31$. However, it should be considered that 19 of 21 patients with EoC-MRD $\geq 5 \times 10^{-4}$ received HSCT versus only three of 20 patients with $\mathrm{MRD}<5 \times 10^{-4}$ (Online Supplementary Figure S6).

We also analyzed the outcome of patients with a WBC higher or less than 100x10\% /L: 5-year EFS was 36.8+12.7\% versus $59.9+11.6 \%$, respectively, $P=0.21$, while 5 -year OS was significantly lower in patients with a WBC $>100 \times 10^{9} / \mathrm{L}(48.8+12.9 \%$ vs. $87.4+6.8 \%, P=0.036)$. This difference was more pronounced in the no-TKI group with a 5 -year EFS $27.8+13.6 \%$ versus $61.8+12.7 \%, P=0.07$ and an OS of $36.7+14.6 \%$ versus $94.4+5.4 \%$, respectively, $P=0.0015)$ (Figure $3 \mathrm{~A}$ and $\mathrm{B}$ ).

\section{Discussion}

The improvement in genetic diagnostics has allowed the identification of rare ALL subgroups with specific clinical characteristics and target lesions. In this context, $\mathrm{ABL}$ class fusion positive B-ALL other than $\mathrm{Ph}^{+} \mathrm{ALL}$ constitutes a challenging entity, which is estimated to represent about $2-3 \%$ of childhood ALL. The frequency is reported in the context of the $B C R-A B L 1-/ \mathrm{Ph}-$ like ALL, which is higher in adolescents and associated with higher risk features and a worse outcome as compared to the non-BCR-ABL1-/Phlike ALL patients. . $^{20,21,23,26}$ First protocols based on "precision medicine" have been designed to identify and treat ALL patients with drugs specific for targetable lesions given in addition to standard chemotherapy (e.g. St Jude Total XVII (NCT03117751) and AALL1131(NCT02883049)). Within the $B C R-A B L 1-/ P h-l i k e$ ALL group, it is suggested that patients with ABL-class fusion positive ALL might benefit from the addition of TKI to chemotherapy. However, besides the above mentioned on-going trials, no controlled studies have been conducted in this field so far, and the published data on the role of TKI for this subgroup is restricted to case reports. ${ }^{21,39,40}$

We report here the largest series of ABL-class fusion positive cases, treated according to two consecutive AIEOP-BFM ALL protocols with a stratification mostly based on MRD response. Screening for ABL-class rearrangements was not required by the protocols and was often done retrospectively in the frame of research projects, thus, no conclusions on incidence can be drawn from this retrospective study. Likewise, due to the selection in screening policy, the outcome data need to be interpreted with great caution. Nevertheless, the cohort described here provides interesting information and clearly shows the urgent need for prospective co-operative clinical studies.

When compared with other B-ALL patients recruited in the AIEOP-BFM ALL protocols, the ABL-class fusion pos- 
itive cases identified in this study included higher proportions of patients aged ten years or older and presenting with hyperleukocytosis (WBC $\left.\geq 100 \times 10^{9} / \mathrm{L}\right)$. Interestingly, the distribution by age and WBC counts is similar to that of patients with $\mathrm{Ph}^{+}$ALL included in the EsPhALL studies. ${ }^{15}$ Poor $M R D$ response at the end of consolidation phase IB $\left(\geq 5 \times 10^{-4}\right)$ was detected in a high proportion of patients with available data (21 of $41,51 \%$ ); similar to that of $\mathrm{Ph}^{+}$ALL patients treated without TKI in the AIEOPBFM ALL 2000 study, where 22 of 54 (41\%) patients had high MRD $\left(\geq 5 \times 10^{-4}\right)$ after consolidation phase. ${ }^{8}$ Interestingly, in patients who had already been treated with a TKI during consolidation, the majority (6 of 8) had either a low positive or negative EoC-MRD, suggesting a beneficial role of the addition of TKI; however, the low number of TKI-treated patients with EoC-MRD data available does not allow any definitive conclusion to be drawn.

The EFS of the entire cohort was poor, particularly for patients not receiving TKI: $<50 \%$ at 5 years, very similar to the outcome of $\mathrm{Ph}^{+}$ALL patients treated in AIEOP-BFM ALL 2000 without TKI. ${ }^{8}$ This poor outcome was observed even though most patients were treated according to the high-risk schedule and more than half of them underwent HSCT in first CR, a strategy similar to that applied for the cohort of $\mathrm{Ph}^{+}$ALL patients of the AIEOP-BFM ALL 2000 study. ${ }^{8}$ Interestingly, the outcome was dismal in patients with $\mathrm{WBC} \geq 100 \times 10^{9} / \mathrm{L}$, as reported also for $\mathrm{Ph}^{+} \mathrm{ALL}$ patients treated in the EsPhALL studies. ${ }^{14,15}$

The impact of HSCT in the cohort reported here is not clear since transplanted patients had an outcome similar to that of patients who were not transplanted. However, patients who received HSCT had worse features, thus, per protocol, an indication for HSCT (and considering the fact that HSCT was associated with a very low rate of relapses), one may infer that HSCT might be effective in disease control. HSCT was, however, also associated with high TRM: six out of 25 patients died of TRM.

Only a minority of our patients (13 of 46) received a TKI (either imatinib or dasatinib). It was not given by protocol; it was used in different schedules, basically decided by treating physicians, and generally due to poor response to chemotherapy. Obviously, the identification of the target lesion had already been achieved during treatment. Of note, most of these patients (9 of 13) also received HSCT, two of them not based on an indication provided by the protocol but on the knowledge of an ABL-class fusion. Although the rate of relapses was low in this small group, their overall outcome was similar to those patients not treated with TKI. Due to the low number of TKI-treated patients, the potentially confounding influence of HSCT and the very short follow up, no conclusions on the benefit of the use of TKI can be drawn from this study. However, given also the biological and clinical similarities with the $\mathrm{Ph}^{+} \mathrm{ALL}$, it is plausible that the early and protracted administration of TKI on top of chemotherapy might improve treatment response and outcome while reducing the need for HSCT in CR1, as shown for $\mathrm{Ph}^{+}$ALL. ${ }^{14-18}$

This study, despite being the largest in this field, is limited by its retrospective nature. The complex interaction of confounding factors, such as case selection bias, stratification criteria, chemotherapy intensity, HSCT, and different timing/modalities of delivering targeted therapies, do not allow the benefit of a precision medicine approach to be appropriately assessed. There is, therefore, an urgent need for a prospective controlled clinical trial.

To this purpose, it will be crucial to include the early identification of ABL-class fusion ALL cases in the initial diagnostic work-up of patients and to treat them in a properly designed study to investigate the role of TKI and to identify the appropriate chemotherapy backbone. Given the rarity of this clinical entity, this goal can only be pursued by an international collaborative network, like in pediatric $\mathrm{Ph}^{+} \mathrm{ALL}$.

\section{Funding}

The project was supported by Grants of Deutsche Krebshilfe, the Madeleine-Schickedanz-Stiftung für Leukämieforschung, the Deutsche Forschungsgemeinschaft (DFG, BE 6555/1-1 and BE 6555/2-1 (GC and AKB)), the Stiftung MHHplus (DSt), the Italian Association for Cancer Research (AIRC) (IG grants to $\mathrm{GiC}$ and $A B)$, the Czech Health Research Council - NV1530626A (MZ) and the Cancer Australia PdCCRs APP1128727 (for RS and DLW). SI and SE were supported by TRANSCALL2 from the Israeli Health Ministry and by the Israeli cancer association.

\section{References}

1. Hunger SP, Mullighan CG. Redefining ALL classification: toward detecting high-risk ALL and implementing precision medicine. Blood. 2015;125(26):3977-3987.

2. Conter V, Aricò M, Valsecchi MG, et al. Long-term results of the Italian Association of Pediatric Hematology and Oncology (AIEOP) Acute Lymphoblastic Leukemia Studies, 1982-1995. Leukemia. 2000; 14(12):2196-2204.

3. Schrappe M, Reiter A, Zimmermann M, et al. Long-term results of four consecutive trials in childhood ALL performed by the ALL-BFM study group from 1981 to 1995. Leukemia. 2000;14(12):2205-2222.

4. Möricke A, Reiter A, Zimmermann M, et al. Risk-adjusted therapy of acute lymphoblastic leukemia can decrease treatment burden and improve survival: treatment results of 2169 unselected pediatric and adolescent patients enrolled in the trial ALL-BFM 95. Blood. 2008;111(9):44774489.

5. Pui CH, Evans WE. Treatment of acute lymphoblastic leukaemia. N Engl J Med. 2006;354(2):166-178

6. Moghrabi A, Levy DE, Asselin B, et al Results of the Dana-Farber Cancer Institute ALL Consortium Protocol 95-01 for children with acute lymphoblastic leukemia. Blood. 2007;109(3):896-904.

7. Carroll WL, Bhojwani D, Min DJ, et al. Pediatric acute lymphoblastic leukemia. Hematology Am Soc Hematol Educ Program. 2003:102-113.

8. Conter V, Bartram CR, Valsecchi MG, et al Molecular response to treatment redefines all prognostic factors in children and adolescents with B-cell precursor acute lymphoblastic leukemia: results in 3184 patients of the AIEOP-BFM ALL 2000 study. Blood. 2010;115(16):3206-3214.
9. Schrappe M, Valsecchi MG, Bartram CR, et al. Late MRD response determines relapse risk overall and in subsets of childhood Tcell ALL: results of the AIEOP-BFM-ALL 2000 study. Blood. 2011;118(8):2077-2084.

10. Bernt KM, Hunger SP. Current concepts in pediatric Philadelphia chromosome-positive acute lymphoblastic leukemia. Front Oncol. 2014;4:54.

11. Fielding AK. Treatment of Philadelphia chromosome-positive acute lymphoblastic leukemia in adults: a broader range of options, improved outcomes, and more therapeutic dilemmas. Am Soc Clin Oncol Educ Book. 2015:e352-359.

12. Aricò M, Schrappe M, Hunger SP, et al Clinical outcome of children with newly diagnosed Philadelphia chromosome-positive acute lymphoblastic leukemia treated between 1995 and 2005. J Clin Oncol. 2010;28(31):4755-4761.

13. Biondi A, Schrappe M, De Lorenzo P, et al. 
Imatinib after induction for treatment of children and adolescents with Philadelphiachromosome positive acute lymphoblastic leukaemia (EsPhALL): a randomised, openlabel, intergroup study. Lancet Oncol. 2012; 13(9):936-945.

14. Biondi A, Cario G, De Lorenzo P, et al. Long-term follow up of pediatric Philadelphia positive acute lymphoblastic leukemia treated with the EsPhALL2004 study: high white blood cell count at diagnosis is the strongest prognostic factor. Haematologica. 2019;104(1):13-16.

15. Biondi A, Gandemer V, De Lorenzo P, et al. Imatinib treatment of paediatric Philadelphia chromosome-positive acute lymphoblastic leukaemia (EsPhALL2010): a prospective, intergroup, open-label, singlearm clinical trial. Lancet Haematol. 2018; 5(12):641-652.

16. Schultz KR, Bowman WP, Aledo A, et al. Improved early event-free survival with imatinib in Philadelphia chromosome-positive acute lymphoblastic leukemia: a children's oncology group study. J Clin Oncol. 2009;27(31):5175-5181.

17. Schultz KR, Carroll A, Heerema NA, et al. Long-term follow-up of imatinib in pediatric Philadelphia chromosome-positive acute lymphoblastic leukemia: Children's Oncology Group Study AALL0031. Leukemia. 2014;28(7):1467-1471.

18. Slayton WB, Schultz KR, Kairalla JA, et al. Dasatinib plus intensive chemotherapy in children, adolescents, and young adults with philadelphia chromosome-positive acute lymphoblastic leukemia: results of Children's Oncology Group Trial AALL0622. J Clin Oncol. 2018;36(22):23062314.

19. Roberts KG, Yang Y, Turner DP, et al. Oncogenic role and therapeutic targeting of ABL-class and JAK-STAT activating kinase alterations in Ph-like ALL. Blood Adv. 2017;1(20):1657-1671.

20. Den Boer ML, van Slegtenhorst M, De Menezes RX, et al. A subtype of childhood acute lymphoblastic leukaemia with poor treatment outcome: a genome-wide classification study. Lancet Oncol. 2009;10(2):125-134.

21. Roberts KG, Li Y, Payne-Turner D, et al. Targetable kinase-activating lesions in $\mathrm{Ph}$ - like acute lymphoblastic leukemia. N Engl J Med. 2014;371(11):1005-1015.

22. Arber DA, Orazi A, Hasserian R, et al. The 2016 revision to the World Health Organization classification of myeloid neoplasms and acute leukemia. Blood. 2016; 127(20):2391-2405.

23. Boer JM, Koenders JE, van der Holt B, et al. Expression profiling of adult acute lymphoblastic leukemia identifies a BCRABL1-like subgroup characterized by high non-response and relapse rates. Haematologica. 2015;100(7):261-264.

24. Harvey RC, Mullighan CG, Wang X, et al. Identification of novel cluster groups in pediatric high-risk B-precursor acute lymphoblastic leukemia with gene expression profiling: correlation with genome-wide DNA copy number alterations, clinical characteristics, and outcome. Blood. 2010; 116(23): 4874-4884

25. van $\operatorname{der}$ Veer A, Waanders E, Pieters R, et al Independent prognostic value of $\mathrm{BCR}$ ABL1-like signature and IKZF1 deletion, but not high CRLF2 expression, in children with B-cell precursor ALL. Blood. 2013;122(15):2622-2629.

26. Roberts KG, Pei D, Campana D, et al. Outcomes of children with BCR-ABL1-like acute lymphoblastic leukemia treated with risk-directed therapy based on the levels of minimal residual disease. J Clin Oncol. 2014;32(27):3012-3020

27. Boer JM, Steeghs EM, Marchante JR, et al. Tyrosine kinase fusion genes in pediatric BCRABL1-like acute lymphoblastic leukemia. Oncotarget. 2017;8(3):46184628.

28. Reshmi SC, Harvey RC, Roberts KG, et al. Targetable kinase gene fusions in high-risk B-ALL: a study from the Children's Oncology Group. Blood. 2017; 129(25):3352-3361

29. Dworzak MN, Buldini B, Gaipa G, et al. AIEOP-BFM consensus guidelines 2016 for flow cytometric immunophenotyping of Pediatric acute lymphoblastic leukemia. Cytometry B Clin Cytom. 2018;94(1):8293.

30. van der Velden VH, Cazzaniga G, Schrauder A, et al. European Study Group on MRD detection in ALL (ESG-MRDALL). Analysis of minimal residual disease by Ig/TCR gene rearrangements: guidelines for interpretation of real-time quantitative PCR data. Leukemia. 2007;21(4):604-611.

31. van der Does-van den Berg A, Bartram CR, Basso $G$, et al. Minimal requirements for the diagnosis, classification, and evaluation of the treatment of childhood acute lymphoblastic leukemia (ALL) in the "BFM Family" Cooperative Group. Med Pediatr Oncol. 1992:20(6):497-505.

32. Reshmi SC, Harvey RC, Roberts KG, et al. Targetable kinase gene fusions in high-risk B-ALL: a study from the Children's Oncology Group. Blood. 2017; 129(25):3352-3361.

33. Basso G, Veltroni M, Valsecchi MG, et al. Risk of relapse of childhood acute lymphoblastic leukemia is predicted by flow cytometric measurement of residual disease on day 15 bone marrow. J Clin Oncol. 2009;27(31):5168-5174.

34. Möricke A, Zimmermann M, Valsecch $M G$, et al. Dexamethasone vs prednisone in induction treatment of pediatric ALL: results of the randomized trial AIEOP-BFM ALL 2000. Blood. 2016;127(17):2101-2112.

35. Kaplan EL, Meier P. Nonparametric estimation from incomplete observations. I Am Stat Assoc. 1958;53(282):457-481

36. Mantel N. Evaluation of survival data and two new rank order statistics arising in its consideration. Cancer Chemother Rep. 1966;50(3):163-170.

37. Kalbfleisch JD, Prentice RL. The statistical analysis of failure time data (ed 1). New York: John Wiley and sons. 1980:163-188.

38. Gray RJ. A class of K-sample tests for comparing the cumulative incidence of a competing risk. Ann Stat. 1988;16(3):11411154

39. Weston BW, Hayden MA, Roberts KG, et al. Tyrosine kinase inhibitor therapy induces remission in a patient with refractory EBF1PDGFRB-positive acute lymphoblastic leukemia. J Clin Oncol. 2013;31(25):413-416.

40 Lengline E1, Beldjord K, Dombret $\mathrm{H}$ Soulier J, Boissel N, Clappier E. Successful tyrosine kinase inhibitor therapy in a refractory B-cell precursor acute lymphoblastic leukemia with EBF1-PDGFRB fusion. Haematologica. 2013;98(11):146148. 